\title{
A Novel Class-Based Protection Algorithm Providing Fast Service Recovery in IP/WDM Networks*
}

\author{
Wojciech Molisz and Jacek Rak \\ Gdansk University of Technology, G. Narutowicza 11/12, \\ Pl-80-952 Gdansk, Poland \\ \{womol, jrak\}@eti.pg.gda.pl
}

\begin{abstract}
In this paper we consider a multilayer architecture: IP-MPLS over optical transport networks (OTNs). Nodes have integrated functionality of optical cross connects (OXCs) in the optical layer and of IP routers in the IP layer. Any two IP routers can be connected together by an all-optical wavelength-division multiplexing (WDM) channel, called a lightpath. Wavelength converters may be installed in each node. We propose a class-based method providing fast service restoration and reducing the number of service recovery actions in the IP layer. We focus on service restoration time values and we show, that it is possible to decrease them, by reducing the size of active path protected area. This may be achieved for the price of link capacity utilization degradation.
\end{abstract}

Keywords: IP /WDM network survivability, multilayer routing, fast, service recovery.

\section{Introduction}

We consider a multilayer architecture: IP-MPLS over optical transport networks (OTNs), the main architecture of the next generation networks [3]. The OTN nodes have functionality of both optical cross connects (OXCs) in the optical layer and of IP routers in the IP layer. Any two IP routers can be connected together by an all-optical wavelength-division multiplexing (WDM) channel, called a lightpath. A lightpath may use several wavelengths if wavelength converters are installed in nodes.

A fundamental characteristic of survivable network systems is their capability to deliver essential services in the face of failure, or attack. There are two approaches for providing survivability of IP-over-WDM networks: protection and restoration [11]. Protection uses pre-computed backup paths applied in the case of a failure. Restoration finds dynamically a new path, once a failure has occurred. In both cases we distinguish either path protection/restoration, or link protection/restoration against a single link or node failure. Several intermediate solutions can be applied between the mentioned extremes, like area protection [7], or partial path protection [13].

* This work was partially supported by the Ministry of Science and Higher Education, Poland, under grants 3 T11D 00130 and N517 022 32/3969. 


\subsection{Related Works}

Recently several papers have appeared on survivability of IP-over-WDM networks. Colle et al. [3] gave an excellent survey of multilayer survivability concepts in the context of next generation networks. Sahasrabuddhe, Ramamurthy and Mukherjee [11], assumming that each backup path in the WDM layer is arc-disjoint with the respective primary path, analysed protection in the WDM layer, and restoration in the IP layer. They proposed four integer linear programming (ILP) models and heuristics to find solutions. Pickavet et al. [8] discussed three approaches to interworking between layers and two efficient coordinated multilayer recovery techniques. Sasaki and $\mathrm{Su}$ [12] proposed models to evaluate the cost of survivable IP-over-WDM networks. Provisioning of survivability at the optical layer provides fast service recovery and is thus inherently attractive, but its major drawback is that it cannot recover from failures that occur at the IP-MPLS layer. The IP layer itself reacts very slowly to failures. Qin, Mason and Jia [9], Lei, Liu and Ji [5], and Ratnam, Zhou and Gurusamy [10] addressed the problem of efficient multilayer operational strategies for survivable IP-over-WDM networks and proposed several joint multiple layer restoration schemes with intralayer and interlayer signaling and backup resource sharing. Zhao et al. [16] proposed a similar strategy for IP/GMPLS/OTN networks. Bigos et al. [2], and Liu, Tipper and Vajanapoom [6] described various methods for spare capacity allocation (SCA) to reroute disrupted traffic in MPLS-over-OTN. Doucette, Grover and Giese [4] used the spare capacity of span-protecting $p$-cycles to provide protection against both optical link and node failures in the IP-MPLS layer.

\subsection{Outline}

We consider here a survivable IP-MPLS-over-OTN-WDM network protected against a single node failure. Demands for IP flows are given. We assume $M$ service classes, numbered from 0 to $M-1$. Class 0 represents the demands for which all the service recovery actions must be performed as fast as possible (i.e. at the WDM layer). For other (lower priority) classes, the IP restoration times may increase.

In the first stage of our algorithm, for each service class we find a node-disjoint working and a backup label switched (LSP) paths. Then we group the IP demands into service classes on IP links. In the next stage we map each class onto protected lightpaths according to available capacities of optical links. The scope of WDM protection depends on service class. For the highest class 0, each two adjacent WDM links of the LSP are protected by a backup lightpath. In contrast, for the lowest class $(M-1)$, there is no backup lightpath and all the recovery actions must be performed at the IP layer. We assume a bottom-up restoration strategy. If an active lightpath consists of more than one link, then a failure of the lightpath transit node can be restored in the optical layer. Connections which cannot be restored in the WDM layer, should be restored in the IP layer. Since all the flow optimization models (in the IP as well as in the WDM layer) are NP-complete, we propose the novel heuristic approach.

The rest of the paper is organized as follows. The survivable routing and wavelength assignment optimization problems are sketched in Section 2. Heuristic algorithms are developed to solve the problems. Modeling assumptions are described in Section 3. Results discussed in Section 4 show, that it is possible to decrease service restoration duration by reducing the size of active path protected area. 


\section{IP-MPLS Survivable Routing of Working/Backup LSPs}

The survivable IP-over-WDM routing problem, is divided into two following subproblems:

a) survivable IP-MPLS routing consisting of determining the IP virtual topology and finding the survivable routing of IP layer demands,

b) survivable WDM routing (lightpath routing and wavelength assignment).

In a subproblem (a) is to find the survivable LSPs, where each working LSP is protected by the end-to-end backup LSP being node-disjoint with the working one. The number of active LSP links depends on the service class $m$ and is determined as:

$$
r_{m}=\left\lceil\frac{l_{c}-1}{M-1} \times m+1\right\rceil
$$

where: $l_{c}$ is the number of links of the end-to-end shortest path between the given pair of source $s$ and destination $d$ nodes in the WDM layer,

$m$ is the class of a demand,

$M$ is the number of service classes.

It is clear from the formula (1), that a working LSP for the class 0 is established by a direct IP layer link. This implies in turn that no IP layer recovery actions will take place. On the contrary, for the class $M-1$ each working LSP link will be mapped onto a single-link WDM lightpath, implying frequent recovery actions at IP layer.

Fast service recovery at WDM layer is achieved here by limiting the backup lightpath protection scope. For that purpose we determine the number of backup lightpaths protecting the given working lightpath as:

$$
b_{m}=\left\lceil-\frac{l_{c}-1}{M-1} \times m+l_{c}-1\right\rceil
$$

where all the symbols have the same meaning as in (1).

From the formula (2), one can observe that $b_{m}$ decreases linearly with the increase of service class number $m$. In particular it means that any backup lightpath for the class 0 protects two adjacent links of the working lightpath, while for class $M-1$ there is no backup lightpath for a given working lightpath and all the recovery actions must be performed in the IP layer. However, due to the limitations on the number of working LSP links in the IP layer given in Eq. 1 (implying the decrease of the length of the working lightpath with the increase of service class number), the real scopes of WDM protection (measured in kilometers of fibers) remain at the same small level, independent of service class number (except for class $M-1$ ). This in turn provides fast service recovery in the WDM layer independent of the service class number.

The proposed approach is characterized by better performance, compared to the approach of solving all the four subproblems separately, since it divides the original problem of integrated IP/WDM routing into two separate subproblems only. Finding the solution to subproblem (a) is followed here by solving the subproblem (b), but the tasks within subproblems (a) and (b) are performed in the integrated manner.

Example IP/WDM routing for demands of boundary classes 0 and $M-1$ is given in Figs. $1 \div 2$. 


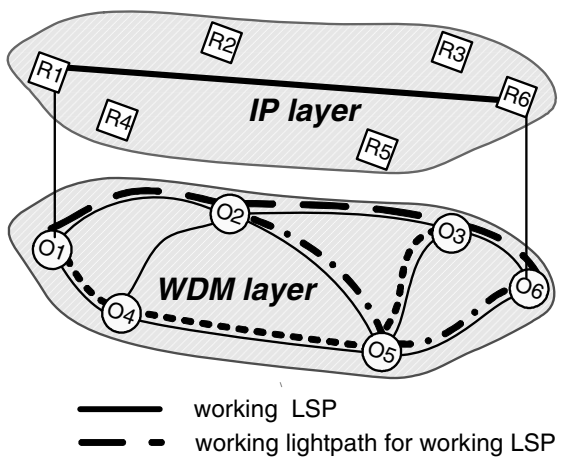

Fig. 1. Example IP/WDM routing of survivable connection of the class $m=0$

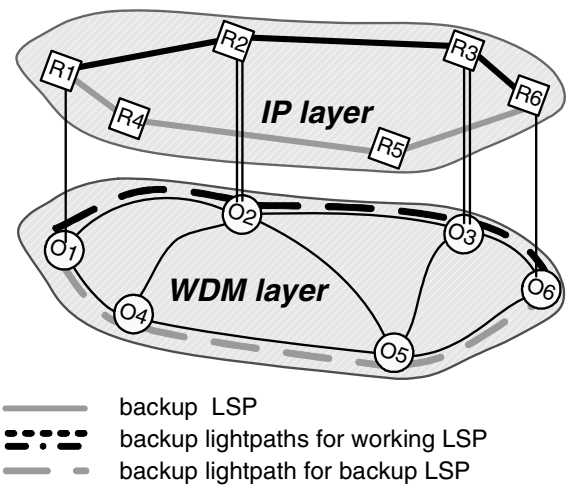

Fig. 2. Example IP/WDM routing of survivable connection of the class $m=M-1$

In the proposed algorithm, given in Fig. 3, the following notation is used:
$s \quad$ - source node of a demand
d - destination node of a demand
$m$ - class of a demand
$\Pi_{s d}$ - the demanded capacity
$\xi_{i j}$ - the cost of the IP link $l_{i j}$ defined as the length of the respective end-to-end lightpath in the WDM layer between nodes $i$ and $j$

\begin{abstract}
INPUT
Network topology; A set of demands, each demand given by a quadruple $\left[s, d, \Pi_{s d}, m\right]$. OUTPUT
\end{abstract}

Survivable multilayer routing of demands from A.

Step 1. Find the working and backup LSPs using the WDM topology with the link costs $\xi_{i j}$ set to link lengths or to infinity in case of insufficient spare capacity.

Step 2. Divide the working LSPs into $r_{m}$ regions, as given in Eq. 1 and replace each working LSP region with a direct IP link. Regardless of the service class $m$, provide each link of the found backup LSP with the one-hop lightpath.

Step 3. Find the lightpaths carrying the IP working flows, using the standard distance metrics treating the aggregated flows belonging to the same class $m$ between the given pair of IP layer nodes, found in Step 2, as the demands for the WDM layer.

Step 4. Divide each working lightpath into $b_{m}$ regions, as given in Eq. 2, and provide each region of WDM lightpath with the dedicated backup lightpath.

Step 5. Provide each aggregated IP backup flow with the unprotected WDM lightpath.

Fig. 3. The proposed algorithm

Steps $1 \div 2$ are responsible for determining the survivable IP-layer routing while steps $3 \div 5$ are used to find the surivivable routing in the WDM layer. Since each of the subproblems considered here is NP-complete, we have used only the heuristic approach in computations.

Due to limitations on the size of this paper, we have put the respective ILP formulations of the above problem at the Web page:

http://www.pg.gda.pl/ jrak/Networking2008_ILP.pdf. 


\section{Modeling Assumptions}

The modeling was performed for the U.S. Long-Distance Network [15], and European COST 239 Network [14], shown in Figs. 4\%5. The simulations were to measure the link capacity utilization ratio, the number of broken connections restored at the IP and WDM layers and the values of connection restoration time.

Time of service restoration in WDM layer comprised: time to detect a failure, link propagation delay, time to configure backup lightpath transit nodes and message processing delay at network nodes (including queuing delay). All the WDM layer links were assumed to have 32 channels. Channel capacity unit was considered to be the same for all the network links. Optical nodes were assumed to have a full wavelength conversion capability. When measuring the IP layer service restoration time, we assumed the IP link propagation delay to be equal to the aggregate delay of the respective WDM lightpath realizing this IP link. The time of message processing delay at the IP layer label-switch routers was assumed to be equal to $30 \mathrm{~ms}$.

For each IP layer connection, the following properties were assumed:

- demands from $M=5$ service classes with protection against a single node failure,

- the demanded capacity equal to $1 / 32$ of the WDM link channel capacity,

- provisioning $100 \%$ of the requested bandwidth after a network element failure,

- a demand to assure unsplittable flows in both IP and WDM layer,

- the distance metrics and Bhandari's algorithm [1] of finding $k$-node disjoint paths (here $k=2$ ) in all path computations, except for the unprotected lightpaths for the backup LSP links, found by the Dijsktra's shortest path algorithm,

- the three-way handshake protocol of service restoration in WDM layer (the exchange of LINK FAIL, SETUP and CONFIRM messages).

The algorithm of a single modeling scenario is shown in Fig. 6.

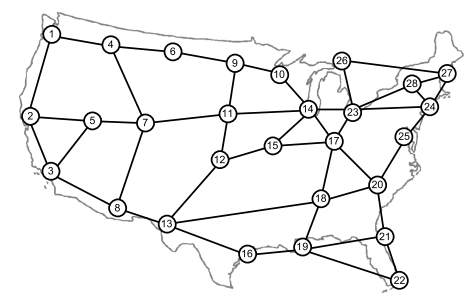

Fig. 4. U.S. Long-Distance Network (28 nodes, 45 bidirectional links)

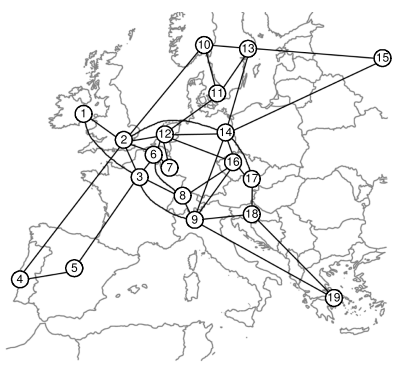

Fig. 5. European COST 239 Network (19 nodes, 37 bidirectional links)

Repeat $r^{*}$ times the following steps:

Step 1. Randomly choose 30 pairs $(s, d)$ of nodes (6 demands for each service class).

Step 2. Try to establish the survivable connections using the proposed algorithm.

Step 3. Store the ratio of link capacity utilization and the lengths of the backup paths.

Step 4. $t^{*}$ times simulate random failures of single nodes. For each failure state restore connections that were broken and store the connection restoration time values.

${ }^{*}$ in each scenario, $r=100$ and $t=100$ were assumed

Fig. 6. Research plan 


\section{Modeling Results}

\subsection{Average Link Capacity Utilization Ratio}

Table 1 shows the average ratios of link capacity utilization achieved during simulation. The amount of capacity needed for backup lightpath purposes turned out to be about two times higher, compared with the amount of capacity needed for active lightpaths. This was due to the fact that additional unprotected lightpaths were needed for backup LSPs to provide protection against the IP layer transit router failures.

Table. 1. Average ratios of link capacity utilization

\begin{tabular}{c|c|c|c} 
Network & $\begin{array}{c}\text { active lightpath } \\
\text { capacity }\end{array}$ & $\begin{array}{c}\text { backup lightpath } \\
\text { capacity }\end{array}$ & $\begin{array}{c}\text { total used } \\
\text { capacity }\end{array}$ \\
\hline U. S. Long-Distance & $3,43 \%$ & $8,60 \%$ & $12,03 \%$ \\
\hline COST 239 & $3,15 \%$ & $7,08 \%$ & $10,22 \%$
\end{tabular}

\subsection{Average Lengths and Numbers of Links of Connection Paths}

Fig. 7 shows the average lengths of IP layer active and backup paths as the function of the service class number, while Fig. 8 gives the respective numbers of IP layer path links. Independent of the service class, the lengths of IP layer active and backup paths remain at the same level, implying similar network resource utilization for all the network classes. The average number of IP layer active path links decreases with the decrease of the service class number (Fig. 8). As a result, time-consuming IP layer recovery actions are less frequent for more important service classes. The average number of IP layer backup path links remains at the same level, independent of the service class, because each IP layer backup path link is established as the one-hop lightpath.

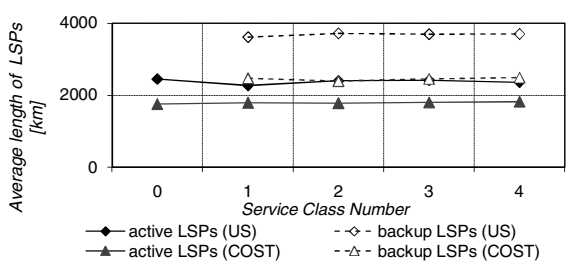

Fig. 7. Average length of paths in the IP layer

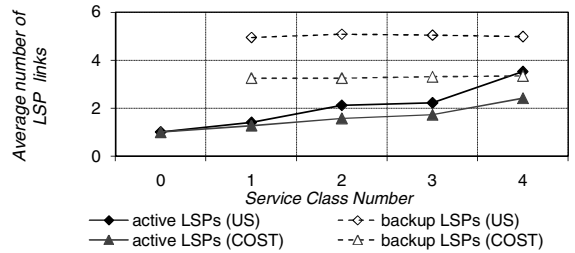

Fig. 8. Average number of LSP links

Fig. 9 shows the average lengths of WDM layer lightpaths as the function of the service class number, while Fig. 10 gives the respective numbers of WDM layer path links. The average length of backup lightpaths remains at the same level, independent of the service class number. However, the average length of active lightpaths decreases with the increase of service class number. This is due to the fact that with the increase of the service class number, the number of IP layer active LSP links increases, and each active LSP link is realized by a shorter WDM lightpath. 


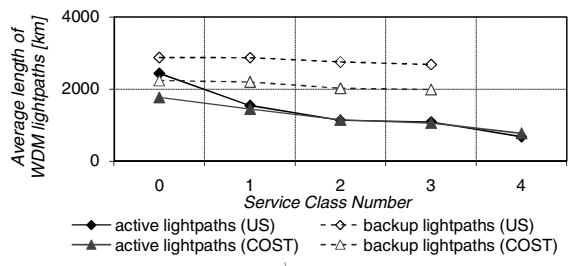

Fig. 9. Average length of WDM lightpaths

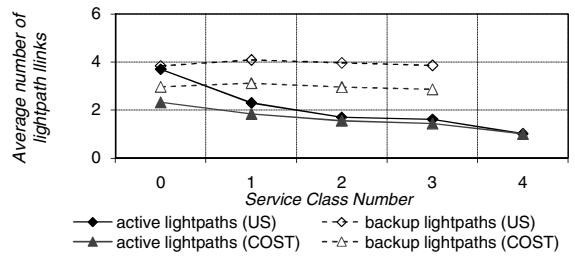

Fig. 10. Average number of lightpath links

\subsection{Service Recovery Actions}

Fig. 11 shows the aggregate numbers of recovery actions for both IP and WDM layer measured in a single scenario. It shows that with the increase of the service class number, the number of recovery actions in IP layer increases, while the number of recovery actions at WDM layer decreases. For the class 0 it implies that WDM layer recovery actions are sufficient to handle all the failure cases and, as a result, provide fast service recovery as given in Fig. 12. For the other service classes, due to the existence of IP layer active path transit nodes, the more IP layer transit nodes the IP active LSP traverses, the higher frequency of IP layer service recovery actions occurs.

The average values of service recovery times in WDM layer remain at the same low level, characteristic to link protection scheme. This is true independent of the service class number, since similar scopes of protection are achieved for all service classes, due to the linear decrease of the number of backup lightpaths that protect a given WDM active lightpath when increasing the service class number, as given in Eq. 2 (i.e. when decreasing the length of WDM active lightpath).

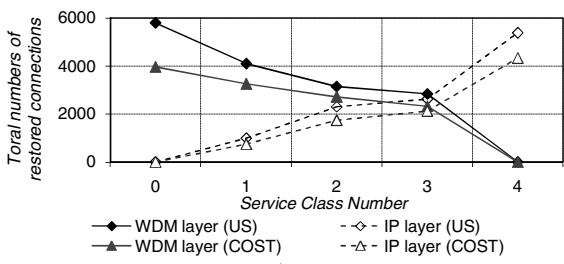

Fig. 11. Total number of restored connections

Fig. 13 shows the aggregate service recovery time values as a function of the service class. Each aggregate value was calculated as the sum of all the values of connection restoration time measured in a single simulation scenario. These results give another proof of the efficiency of our approach. For the U.S. Long-Distance Network the ratio between classes 0 and 4 is of order 1:6, while for the European COST 239 Network - about 1:6.6. Aggregate restoration times for the highest (0) class are always the shortest ones.

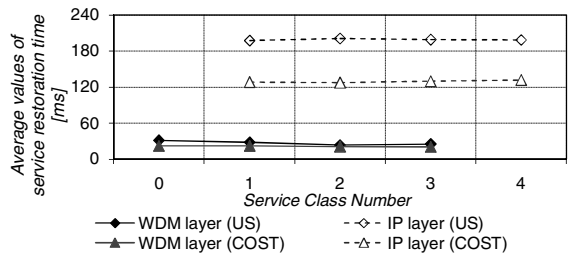

Fig. 12. Average service recovery time values

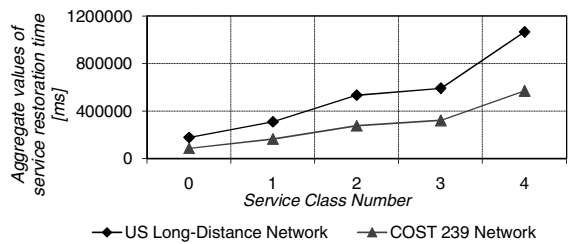

Fig. 13. Aggregate values of service restoration time 


\section{Conclusions}

In this paper we considered the multilayer architecture of the Next Generation Networks: IP-MPLS over OTN-WDM. We assumed nodes having the integrated functionality of optical cross connects (OXCs) and of IP routers in the IP layer. We proposed a class-based method providing fast service restoration and reducing the number of service recovery actions in the IP layer. We focused on service restoration time values and we showed, that it is possible to decrease them, by reducing the size of active path protected area. To prove our thesis, we modeled two typical networks: the U.S. Long-Distance Network and the European COST 239 Network and have calculated: the link capacity utilization ratio, average lengths and numbers of links of the connection paths and the values of service restoration time. To the best of our knowledge, all results are original ones and published for the first time.

\section{References}

1. Bhandari, R.: Survivable networks: Algorithms of diverse routing. Kluwer Academic Publishers, Boston (1999)

2. Bigos, W., et al.: Survivable MPLS over Optical Transport Networks: Cost and Resource Usage Analysis. IEEE J. Select. Areas Commun. 25(5), 949-962 (2007)

3. Colle, D., et al.: Data-Centric Optical Networks and Their Survivability. IEEE J. Select. Areas Commun. 20(1), 6-21 (2002)

4. Doucette, J., Grover, W.D., Giese, P.A.: Physical-Layer p-Cycles Adapted for Router Level Node Protection: A Multi-Layer Design and Operation Strategy. IEEE J. Select. Areas Commun. 25(5), 963-973 (2007)

5. Lei, L., Liu, A., Ji, Y.: A joint resilience scheme with interlayer backup resource sharing in IP over WDM networks. IEEE Communications Magazine 42, 78-84 (2004)

6. Liu, Y., Tipper, D., Vajanapoom, K.: Spare Capacity Allocation in Two-Layer Networks. IEEE J. Select. Areas Commun. 25(5), 974-986 (2007)

7. Molisz, W., Rak, J.: Region Protection/Restoration Scheme in Survivable Networks. In: Gorodetsky, V., Kotenko, I., Skormin, V.A. (eds.) MMM-ACNS 2005. LNCS, vol. 3685, pp. 442-447. Springer, Heidelberg (2005)

8. Pickavet, M., et al.: Recovery in Multilayer Optical Networks. IEEE J. of Lightwave Technology 24(1), 122-133 (2006)

9. Qin, Y., Mason, L., Jia, K.: Study on a joint multiple layer restoration scheme for IP overWDM networks. IEEE Network 17(2), 43-48 (2003)

10. Ratnam, K., et al.: Efficient Multi-Layer Operational Strategies for Survivable IP-overWDM Networks. IEEE J. Select. Areas Commun. 24(8), 16-31 (2006)

11. Sahasrabuddhe, L., et al.: Fault management in IP Over WDM Networks: WDM Protection vs. IP Restoration. IEEE J. Select. Areas Commun. 20(1), 21-33 (2002)

12. Sasaki, G.H., Su, C.-F.: The Interface between IP and WDM and Its Effect on the Cost of Survivability. IEEE Communications Magazine 41, 74-79 (2003)

13. Wang, H., Modiano, E., Médard, M.: Partial Path Protection for WDM Networks: End-toEnd Recovery Using Local Failure Information. In: Proc. ISCC 2002, vol. 725, pp. 719725 (2002)

14. Wauters, N., Demeester, P.: Design of the optical path layer in multiwavelength cross connected networks. IEEE J. Select. Areas Comn. 1(5), 881-892 (1996)

15. Xiong, Y., Mason, L.G.: Restoration strategies and spare capacity requirements in self healing ATM networks. IEEE/ACM Trans. on Netw. 7(1), 98-110 (1999)

16. Zhao, J., et al.: Integrated multilayer survivability strategy with inter-layer signaling. In: Proc. of Int. Conf. on Communication Technology, ICCT 2003, vol. 1, pp. 612-616 (2003) 